\title{
Speaker Recognition System Based on the Baseband Correlation Score Reliability Fusion
}

\author{
Qi He ${ }^{1}$, Ting Huang ${ }^{2}$, Hongbo Zhang ${ }^{3 *}$ \\ ${ }^{1}$ Science and Technology Department of Ningxia, Yinchuan, China \\ ${ }^{2}$ MicroStrategy Software (Hangzhou) Co., Ltd., Hangzhou, China \\ ${ }^{3}$ School of Physics \& Electrical Information Engineering, Ningxia University, Yinchuan, China \\ Email: *904291161@qq.com \\ Received May 2013
}

\begin{abstract}
Emotion mismatch between training and testing will cause system performance decline sharply which is emotional speaker recognition. It is an important idea to solve this problem according to the emotion normalization of test speech. This method proceeds from analysis of the differences between every kind of emotional speech and neutral speech. Besides, it takes the baseband mismatch of emotional changes as the main line. At the same time, it gives the corresponding algorithm according to four technical points which are emotional expansion, emotional shield, emotional normalization and score compensation. Compared with the traditional GMM-UBM method, the recognition rate in MASC corpus and EPST corpus was increased by $3.80 \%$ and $8.81 \%$ respectively.
\end{abstract}

Keywords: Emotional Speaker Recognition; Pitch Normalization Method; Model Mismatch Detection; Emotional Normalization

\section{Introduction}

Because of the inconsistent training and testing conditions, the model of the training set cannot effectively describe the characteristic distribution of the test voice, referred to herein as the model mismatch. The core of speaker recognition technology is the pre-entry of the speaker's voice samples to which is extracted as the unique speaker voice feature and stored in the database, according to match the testing speech with the characteristics in database, then determine the identity of the speaker. There are many factors that affect the performance of the speaker recognition system in a real environment; their sources can be divided into external factors and internal factors. External factors mainly come from the ambient noise, channel change and the difference of coding scheme. Internal factors change, also known as intra-Speaker variation, refers to the speaker's own physiological characteristics or personality and behavior characteristics change, can be divided into the two categories of long-term and short term. The long-term change [1] generally refers to the vocal organs slow changes due to the increasing age of the speaker. The short-term disease generally refers to the temporary illness such as cold, cough, inflammation of the tonsils, inflammation of the gums which could cause the change of vocal organs.

\footnotetext{
"Corresponding author.
}

Emotional change is another common factor to cause performance degradation of speaker recognition system. Different emotional statuses cause the different effect of speaker's utterance mechanism. This will lead to the change of the personality traits of the speaker's voice, and then lead to the mismatch between training and testing feature space distribution.

We propose a score reliability fusion based on the baseband correlation to avoid identifying the user's specific emotional type. This method using the correlation of baseband mean deviation and the recognition rate existence between high difference emotional speech and neutral voice, by reducing the weight of high-baseband mean deviation part in high difference emotional speech, to reduce the score of impersonate model and improve the score of the real speaker model. Thus, the performance for speaker recognition is improved.

\section{The Application and Mismatch Effects of the Baseband in Speaker Recognition}

The voice is the result of the combined effect of the sound source and channel, sound source excitation signal, by modulation of channel and radiation effects of nose and mouth, form the final speech. The speech contain two parts (the sound source and channel) [2] of information. In phonetics, we usually use cepstral coefficients 
(such as MFCC, LPCC [3]) to describe the speech signal channel response. And use pitch frequency to describe the excitation of the speech signal sound source. Using global statistics of baseband can enhance the performance of speaker recognition. But because of the lack of description of the baseband partial information, the existing methods are generally by speech segmentation, then extract statistical features to make up for the shortfall in the baseband fragment.

As the speech baseband characteristics contains the speaker's personality traits, and characteristics of no effect by the channel and noise. So it has been used to improve system robustness of channel mismatch and environmental noise in speaker recognition. However, in the actual environment, the baseband is often affected by the change of text content of the speaker's voice, manner of speaking and emotional state. Thus, in these circumstances, using the difference of speaker baseband feature to distinguish the speaker performance will be greatly reduced. The mismatch of baseband distribution between training and testing by the same speaker is baseband mismatch [4]. It would lead a negative impact to speaker recognition. Emotional change is one of the main factors leading to baseband mismatch. The shape of speech baseband envelope curve is dependent on the speaker's speech emotional state [5].

It is different from external factors such as ambient noise, channel mismatch. The most obvious performance of emotional changes in speech is the change of the prosodic features such as baseband. So it is not feasible to take baseband relevant information as the characteristics of emotional speaker recognition. On the basis of indepth study of prosodic features such as baseband in the emotional changes and Interference phenomena between baseband characteristics and channel characteristics (MFCC), in this article, we proposed several emotions compensation algorithm to reduce the effect of speaker's emotional state mismatch between training and testing to the speaker recognition system.

\section{Pitch Normalization Method (PNM)}

As it was found there was severe deviation in pitch mean between HMS and corresponding neutral speech, the idea of PNM was to normalize pitch of HMS more approximately to the neutral speech. Following the thought, firstly, varied proportion of pitch mean between HMS and corresponding neutral speech was need to obtain. The proportion $F$ was defined as follow:

$$
F=\bar{H} / \bar{L}
$$

In Equation (1), $\bar{H}$ and $\bar{L}$ were pitch mean of the HMS and of the responding neutral speech respectively. Because speaker was unknown in testing, $\bar{L}$ was unknown. It was assumed that the function mapping relation $f_{g}$ mapped $\bar{H}$ to $\bar{L}$, i.e.:

$$
\bar{L}=f_{g}(\bar{H})
$$

The subscript $g$ presented gender, because $f_{g}$ was related with speaker's gender. Equation (1) turned to Equation (3):

$$
F_{g}=\bar{H} / \bar{L}=\bar{H} / f_{g}(\bar{H})
$$

And then pitch of HMS was normalized by the following equation:

$$
L_{t}=H_{t} / F_{g}=H_{t} /\left(\bar{H} / f_{g}(\bar{H})\right)
$$

$H_{t}$ was the pitch of a period of emotional speech, while $L_{t}$ was the normalized pitch that approximated the speaker's corresponding neutral speech.

Obviously, the form of $f_{g}$ was unknown and hard to solve analytically. Polynomial was a smooth and continuous function, and its differential form was also a polynomial. So it was a good choice for polynomial to fit $f_{g}$. Polynomial form was as follow:

$$
f_{g}(x)=\sum_{i=0}^{k_{g}} a_{i g} x^{i}
$$

Polynomial order $k_{g}$ was able to obtain by Akaike information criterion (AIC) [1]. One of AIC forms was adopted here as follow:

$$
A I C=2\left(k_{g}+1\right)+n[\ln (R S S / n)]
$$

In Equation (6), $n$ was sample count, and RSS was residual sum of squares (RSS). When AIC got its minimum value, corresponding $k_{g}$ got its most proper order for the polynomial. Then the method of least squares (LS) was able to solve polynomial coefficients $a_{i g}$, i.e., $f_{g}$ was fit by polynomial.

\section{Score Reliability Fusion Based on the Baseband}

In this paper, we propose a score reliability fusion based on the baseband, getting the voice contribution to correct identification voice by way of evaluating high-mismatch parts of speech, and taking the contribution as the frame score weighting weights of high-mismatch parts of speech in test. Finally, we achieve an effective use of speech high-mismatch parts.

\subsection{Score Reliability Fusion Based on the Baseband Correlation}

In speaker recognition, every frame in test voice makes a contribution to correctly identify. When the frame plays a positive role for the correct identification, contribution is positive, otherwise it is negative. When test voice is incorrectly identified, contribution of a part of frame score must be negative. Different frame score contribution in the speech frame level using different weights. It is ob- 
vious to improve the score of the real speaker and reduce the score of impersonation. Thus, the performance for speaker recognition is improved.

According to the study of the relationship between the baseband mean deviation and the degree of mismatch, for HD emotional speech. The greater the baseband mean deviation, the lower speaker recognition rate is. That is, the more unreliable the score calculated is, and the smaller the contribution for a system to correctly identify is. So, we propose a score reliability fusion based on the baseband deviation correlation of difference determine, specific processes shown in Figure 1. The method to obtain the weighting coefficients can be divided into four steps:

1) Build reliability fusion coefficients related to baseband for male and female speaker respectively;

2) Use gender recognition to distinguish Gender information of test speech;

3) Detect the High Mismatch Segment (HMS) of testing speech according to the method in Chapter 5;

4) Calculate the score weighting coefficients of each of the test speech frame, according to the high mismatch identification and reliability weighting coefficient.

\subsection{High Mismatch Detection}

There is a discontinuity in the speaker's emotional expression. Even in the same speech, there are some fluctuations. The purpose of the method is to identify the serious mismatch segment relative to the neutral model in speech. That is high mismatch segment. And reduce its weight when calculating the final test statement score, in order to improve the speaker recognition performance.

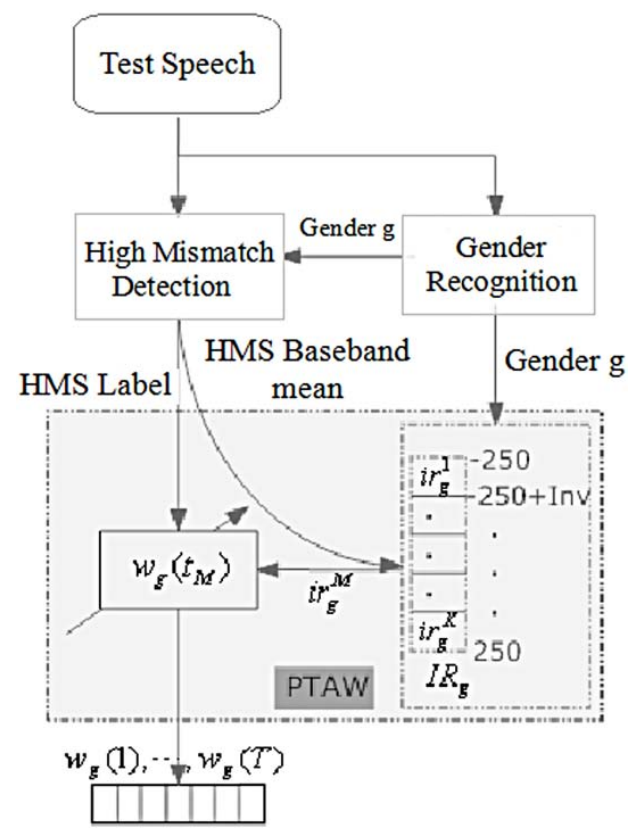

Figure 1. System flowchart of score reliability fusion based on the baseband correlation.
For high mismatch detection, the first step is to detect whether the testing speech belongs to the high difference emotional speech, by using difference detection method fusion of the short acoustic characteristics and statistical prosodic features. Then divide the testing speech into several baseband snippets. The next, the baseband mean which is higher than the threshold (male: $156 \mathrm{~Hz}$, female: $250 \mathrm{~Hz}$ ) is marked as the high mismatch segment.

\subsection{Score Reliability Fusion Function Based on the Baseband Correlation}

For high difference emotion, there is a positive correlation between baseband mean deviation and the degree of mismatch of emotional state. In this article, we use the correlation to build a score reliability fusion function related to baseband for high mismatch segment. But for low mismatch segment, we consider it is relatively close to its neutral, and its frame score weight is set as 1 .

We use the deviation between tested voice baseband mean and the groups of neutral voice baseband to instead that deviation between tested voice baseband mean and corresponding neutral voice-based frequency deviation from the mean. In addition, voice baseband deviation band speaker voice recognition as one of the band's neutral voice matches the State, that is, the coefficient of reliability weighted scores on such frames. Below we define specific forms of the weighting function:

1) We concentrated frequency values $F_{g}$ of neutral voice fundamental which is in set of parameters developed ( $g$ is the gender), and deviate speaker's baseband of the mean of range $[-250,250]$ into k equal parts, each of equal parts is $I n v \ll 500 / K$;

2) We calculate the identification rate $\operatorname{IR}_{\mathrm{g}}^{\mathrm{m}}$ of set of parameters developed male and female high difference class Emotional Speech which are among the every deviation interval $R_{m}(m \ll 1,2, \ldots, K)$.

3) For a period of test speech $X \ll\left\{x_{t} \mid t \ll 1,2, \Lambda, T\right\}$, the Score reliability weighting function is:

$$
w_{g}(t)=\left\{\begin{array}{cc}
e_{m(t)} I R_{g}, & \text { if } t \in S_{H M S} \\
1 & \text { else }
\end{array}\right.
$$

We should know

$$
e_{i}=\underbrace{[0, \cdots, 1, \cdots, 0]}_{M}
$$

$m(t)$ is the trame pitch deviation of mean value relative to the base frequency of the pieces fall within the $\mathrm{m} / \mathrm{K}$ range, $I R_{g} \ll\left[\mathrm{ir}_{\mathrm{g}}^{1}, \ldots, \mathrm{ir}_{\mathrm{g}}^{\mathrm{K}}\right]^{T}$ is the high differences class of emotional speech recognition rate that we precompute the gender related deviation band in step 2. $S_{H M S}$ is the frame number collection of the high mismatch in the testing speech. When the frame score weighting factor in $S_{H M S}$ collection is zero, we call that excluding strategy. 


\subsection{Speaker Recognition System of Score Reliability Fusion Based on the Baseband Correlation}

Speaker recognition model training is the same as the traditional speaker recognition. For the collection of $\mathrm{N}$ registered speaker, we train one speaker model $\lambda_{i}$ for every speaker, $1 \leq i \leq N$. In the test, we should determine the gender information of testing speech $X \ll\left\{x_{t} \mid \mathrm{t} \ll 1,2\right.$, $\Lambda, T\}$, then calculate the reliability weighting coefficients $w_{g}(t), t \ll 1,2, \Lambda, T$. At last, we have a weight for $X$ about the score for each frame of $\Lambda \ll\left\{\lambda_{1}, \lambda_{2}, L, \lambda_{N}\right\}$, and the testing speech is determined the speaker i corresponded the probability value of the maximum model.

$$
\mathrm{i}^{*}=\arg \max _{1 \leq i \leq N} W \log P\left(X \mid \lambda_{i}\right)=\arg \max _{1 \leq i \leq N} \sum_{t=1}^{T} w_{g}(t) \log p\left(x_{t} \mid \lambda_{i}\right)
$$

\section{Experimental Analysis and Discussion}

Experimental corpus base Mandarin Affective Speech Corpus (MASC) and Emotional Prosody Speech and Transcripts (EPST). MASC has 23 female and 45 male speakers' utterance in Chinese mandarin with 5 emotional classifications (neutral, angry, happy, scared, and sad classifications). Every speaker has 5 phrases and 60 sentences in every emotional state. Each phrase lasts 0.8 second averagely, while each sentence lasts 2 seconds averagely. Besides, there are 2 short passages with average duration of 15 seconds per passage in neutral state. EPST is the first emotional speech corpus released by Linguistic Data Consortium (LDC). It includes 8 actors (3 male, 5 female). 7 speakers of them provide their English speech in 14 emotional classifications and their neutral speech with different distance. The corpus used in the experiment is split into 3 parts: Speeches of the first 18 people ( 7 female and 11 male) in MASC were taken as development data to obtain fitting parameters; Speeches of the remains in MASC were test data. 2 short passages of every speaker were used to train speaker model, and the other 15,000 sentences were used as testing speeches; In addition, speeches of 7 speakers in EPST corresponding with 5 same emotional classifications as MASC were treated as extended test data. About 5 minutes neutral speeches of each speaker in normal distance were used to train speaker model. 5 kinds of emotional sentences with total count 670 were taken as testing speech.

In the experiment, UBM is adopted 1024 orders and characteristics are 13-dimensional MFCC and its delta. The length of window for MFCC, energy and pitch are $32 \mathrm{~ms}$ uniformly, and step sizes are $16 \mathrm{~ms}$ uniformly. All neutral speeches of the first 18 people in MASC were used as training speeches for UBM, and UBM was obtained by expectation maximization algorithm (EM). For every speaker, his speaker model was obtained using his neutral speech from UBM by MAP. In addition, GMM is adopted 1024 orders in gender recognition.

For verifying the validity of two kinds fusion weight estimating strategy based on the score reliability assessment, this part will compare the four methods of recognition performance on the MASC corpus and EPST corpus. The four methods are: the bi-model method fusion weight estimating strategy based on the score reliability assessment (score difference), the bi-model method based on the weight strategy of recognition rate (recognition rate), the bi-model method based on the equal weight (equal weight) and the traditional GMM-UBM method (datum).

\subsection{Experimental results on the MASC}

For each speaker, the two-stage neutral paragraphs speech are used to each speaker models from the UBM adaptive. The specific experimental results of the three methods are shown in Table 1. From the table, we first found this method as opposed to elimination in anger, pleasure and panic on the recognition rate improved, $0.7 \%, 2.00 \%$ and $1.53 \%$ respectively. This suggests that high mismatch part which is excluded of your tests to correctly identify the voice still has a role, and recognition of the baseband-weighted approach can effectively measure the role of that part of the speech. For two emotion of LD type, neutral and sad, due to difference testing had been wrongfully convicted as a high difference in emotional, leading to more significant decrease in the elimination. The LD type emotional statement which is wrongfully convicted, identified as highly mismatched parts of voice baseband is relatively low. Weights in weighted method in this paper is much higher, thereby avoiding removal method to ignore the part of speech, negative impact on system performance. Relative to the elimination method in this article as a whole has increased $1.1 \%$, relative to the base has increased $2.48 \%$.

\subsection{Experimental Results on the EPST}

The EPST corpus and MFC corpus are the speech database under two different cultural backgrounds. To further validate the effectiveness of the proposed method, we

Table 1. Experimental results on the MASC.

\begin{tabular}{cccc}
\hline \multirow{2}{*}{ Method } & \multicolumn{3}{c}{ IR (\%) } \\
\cline { 2 - 4 } & Datum & Reject & Baseband weight \\
\hline Neutral & 96.23 & 95.47 & 96.13 \\
Angry & 31.50 & 35.80 & 36.50 \\
Happy & 33.57 & 36.40 & 38.40 \\
Scared & 35.00 & 36.10 & 37.63 \\
Sad & 61.43 & 60.87 & 61.50 \\
Average & 51.55 & 52.93 & 54.03 \\
\hline
\end{tabular}


Table 2. Experimental results on the EPST.

\begin{tabular}{cccc}
\hline \multirow{2}{*}{ Method } & \multicolumn{3}{c}{ IR (\%) } \\
\cline { 2 - 4 } & Datum & Reject & Baseband weight \\
\hline Neutral & 93.75 & 93.75 & 93.75 \\
Angry & 47.48 & 46.76 & 48.20 \\
Happy & 39.62 & 49.06 & 45.92 \\
Scared & 39.72 & 53.19 & 51.77 \\
Sad & 66.89 & 69.54 & 70.86 \\
Average & 53.88 & 59.40 & 58.96 \\
\hline
\end{tabular}

have a compare between recognition performance of the method and the traditional method on the EPST corpus. The method in EPST corpus in addition to the four neutral emotional speech recognition performance has improved $0.72 \%$ to $12.05 \%$. But we also found that, there are large differences speaker's emotional expression between the presence in the EPST corpus and MASC corpus, and the reliability weighting coefficient based on the baseband learned from MASC can't describe the score contribution of the each baseband region of high mismatch portion in the EPST corpus well. Therefore the recognition rate under the happy and sad two emotions is not as good as the direct effect of excluding (Table 2).

\section{Conclusion}

Due to the baseband mean deviation of the neutral speech for high different emotional speech has the correlation with recognition rate. This paper proposes the related score reliability weighting system based on the baseband. This method is to reduce the score on entire test voice impersonate model and improve their score on the target speaker model by reducing the weight of high-frequency in high different emotion speech. As a result, the recognition performance can be improved. Experimental results on MASC corpus show that, the method increases the recognition rate by $2.48 \%$ relative to the traditional GMM-UBM. In addition, in the EPST corpus, the 5.08\% increase compared to the traditional GMM-UBM.

\section{Acknowledgements}

This research was supported by the Natural Science Foundation of Ningxia Hui Autonomous Region, China (Grant No. NZ1139), and Scientific and technological projects in Ningxia (The research and development application demonstration of Ningxia milk and the products' safety traceability information system which is based on the Internet of Things). All supports are gratefully acknowledged.

\section{REFERENCES}

[1] M. Pawlewski and J. Jones, "URU Plus-A Scalable Component-Based Speaker-Verification System for BT's 21st Century Network,” BT Technology Journal, Vol. 25, No. 3-4, 2007, pp. 170-178. http://dx.doi.org/10.1007/s10550-007-0072-y

[2] J. Q. Han, L. Zhang and Y. R. Zheng, "Speech and Signal Processing,” Tsinghua University Press, Beijing, 2004.

[3] S. Furui, "Cepstral Analysis Technique for Automatic Speaker Verification,” IEEE Transactions on Acoustics, Speech and Signal Processing, Vol. 29, No. 2, 1981, pp. 254-272. http://dx.doi.org/10.1109/TASSP.1981.1163530

[4] R. D. Zilca, B. Kingsbury, J. Navratil, et al., "Pseudo Pitch Synchronous Analysis of Speech with Applications to Speaker Recognition," IEEE Transactions on Audio Speech and Language Processing, Vol. 14, No. 2, 2006, pp. 467-478. http://dx.doi.org/10.1109/TSA.2005.857809

[5] D. Morrison, R. Wang and L. C. De Silva, "Ensemble Methods for Spoken Emotion Recognition in Call-Centres,” Speech Communication, Vol. 49, No. 2, 2007, pp. 98-112. http://dx.doi.org/10.1016/j.specom.2006.11.004 